\title{
Transcutaneous Electrical Nerve Stimulation (TENS) for Primary Dysmenorrhea: An Overview
}

This article was published in the following Dove Press journal:

International Journal of Women's Health

\section{Michal Elboim-Gabyzon (ID) Leonid Kalichman (1D) ${ }^{2}$}

'Physical Therapy Department, Faculty of Social Welfare and Health Sciences, University of Haifa, Haifa 2242I, Israel; ${ }^{2}$ Department of Physical Therapy, Recanati School for Community Health Professions, Faculty of Health Sciences, Ben-Gurion University of the Negev, Beer Sheva, Israel
Correspondence: Michal Elboim-Gabyzon Physical Therapy Department, Faculty of Social Welfare and Health Sciences, University of Haifa, Balfour 9 Nahariya, Haifa 2242I, Israel

Tel +972-545968943

Email michal.elboim@gmail.com
Abstract: Primary dysmenorrhea is a chronic health condition that affects primarily young women. Transcutaneous electrical nerve stimulation (TENS) has been suggested as an effective pain reduction modality in primary dysmenorrhea. TENS is a noninvasive, inexpensive, portable method with minimal risks and a few contraindications. When necessary, it can be self-administered on a daily basis during everyday activities. Several studies have investigated the effectiveness of TENS in reducing pain, decreasing the use of analgesics, and improving the quality of life in primary dysmenorrhea patients. These studies have some limitations in methodological quality and therapeutic validation. However, the overall positive effects of TENS in primary dysmenorrhea encountered in all prior studies indicated its potential value. This review presents the clinical recommendations for TENS parameters for treating primary dysmenorrhea symptoms based on previously published studies.

Keywords: primary dysmenorrhea, transcutaneous electrical nerve stimulation, TENS, pain, life quality, treatment

Primary dysmenorrhea (PD) is the most common gynecological symptom reported by women of reproductive age. ${ }^{1}$ It is defined as the recurrent, cramping pain, which occurs during menses, and lacks any identifiable reproductive system pathology. ${ }^{1,2}$ While cramping pain may be the only complaint, one or more of the following symptoms may accompany the pain: nausea, breast tenderness, diarrhea, fatigue, headache, dizziness, and more rarely, syncope and fever. ${ }^{1} \mathrm{PD}$ is usually treated by pharmacological agents that are often accompanied by adverse side effects. ${ }^{3,4}$ Accordingly, integrating alternative nonpharmacological, noninvasive analgesia is preferred.

One of the noninvasive treatment methods is transcutaneous electrical nerve stimulation (TENS) that delivers electrical currents through the skin. High-frequency (HF) TENS was demonstrated to be effective in reducing menstrual pain in a 2002 Cochrane review of seven previously published studies by Proctor et $\mathrm{al}^{4}$ This review was updated in 2009 without the addition of new studies. ${ }^{4}$ Since the 2009 update, ${ }^{4}$ six new trials had been published that further support HF TENS as an effective treatment modality for PD. ${ }^{5-10}$ Additionally, two recent reviews ${ }^{1,11}$ and a systematic review on the management of $\mathrm{PD}^{12}$ advocated TENS as a safe and effective method for pain reduction. However, the review of the literature indicates that information on application parameters and clinical considerations regarding the use of TENS in PD is still lacking. In the present study, we attempted to provide the best available data on the pathophysiology and mechanism of action of TENS in dysmenorrhea, the best clinical recommendations for the TENS parameters, and interactions with pharmacological agents. 


\section{The Pathophysiology and Mechanism of Action of TENS in PD}

The pathophysiology of PD should be considered when dealing with some of the variables of TENS, such as the optimal timing and duration of treatment. Endocrine factors have been suggested because PD occurs only during ovulatory cycles. Recent studies have indicated excessive secretion of prostaglandins and decreased ovarian steroid hormone levels in the endometrium as causes of PD. ${ }^{2,13}$ These changes result in hypercontractility of the uterus that lasts for hours with possible elevated intrauterine pressure $^{1}$ and decreased blood flow, and leads to uterine hypoxia and ischemia, which are believed to cause the underlying etiologies for the pain and cramps. ${ }^{11}$

Vasodilation, increased blood flow, and menstrual fluid prostaglandins may be altered by TENS, and may indirectly induce analgesic effects. These effects are probably mediated by the inhibition of nociceptive fiber-evoked responses in the dorsal horn. Based on the gate control theory of pain, this inhibition is caused by stimulation of large diameter afferents which are stimulated by TENS. ${ }^{15}$ Sensory stimulation of the skin may also cause local vasodilation in the same dermatome via axonal reflexes. ${ }^{16}$ The effects of TENS are also modulated by anatomic pathways, ${ }^{17}$ opioid release, ${ }^{24}$ decreased release of prostaglandins, and possibly eicosanoids that are released from the endometrium during menstruation ${ }^{25}$ following antidromic blockage of nerve fibers with large diameters. ${ }^{26}$

\section{Design of the Reviewed Studies}

Previous studies adopted a variety of approaches, including the comparison of HF TENS versus placebo, ${ }^{6,7,10,17-19}$ low-frequency (LF) TENS versus placebo, ${ }^{16,17,19,20} \mathrm{HF}$ versus LF TENS, ${ }^{17,19}$ and TENS versus medication. ${ }^{3,5,18,19}$

One study lacked a placebo group and each participant was used as her own control. ${ }^{8}$ Another study compared HF TENS and interferential current. ${ }^{9}$ The studies used parallel $^{6,7,9,16,17,20}$ or crossover designs..$^{3,8,10,18,19}$

Many previous trials were characterized by the lack of consistency with respect to the study design and homogeneity of the participants. The women had different demographic and physical characteristics (including their ages, heights, and weights), menstrual history, severity of the condition, and in particular, there were differences in pain levels, general physical activity levels, function, and quality of life. Furthermore, the trials exhibited increased degrees-of-variation in the use of TENS in terms of dose (current intensity and frequency, duration, and frequency of delivery), the location of the electrodes, blinding, and the control group. Moreover, one of the trials reported on the efficiency of TENS combined with ibuprofen on pain reduction, but important details on the dose were not disclosed. ${ }^{5}$

\section{Guidelines for the Application of Sensory-Level TENS for PD}

TENS requires setting many parameters related to current and electrode characteristics. Table 1 and Figure 1 summarize the clinical recommendations, and provide an overview of the parameters and considerations based on previous studies. Some critical points will be discussed hereinafter.

\section{TENS Current Frequency}

Sensory-level TENS frequencies are classified in two types: 1) HF (>50 Hz) or 2) LF $(2-5 \mathrm{~Hz}) .{ }^{21} \mathrm{HF}$ and LF mainly differ with regard to the type of sensory fibers that the current activates. HF activates large diameter, non-noxious cutaneous afferent (A $\beta)$ mechanoreceptors, while the small diameter nerves are not stimulated (such as c fibers, muscle efferent, or A $\delta$ fibers). HF is used more often. Accordingly, it is referred as conventional TENS, and is applied at low intensities. The pattern of pulse delivery is usually continuous, although it can be delivered in "bursts" or "trains." 22 The patient experiences an intense, comfortable, nonpainful paresthesia over the site of pain dermatomes. LF TENS consists of high-intensity pulses delivered in a range of frequencies from $1 \mathrm{~Hz}$ to $4 \mathrm{~Hz}$, and activates motor efferent nerves to produce phasic muscle twitches. These events ultimately lead to the activation of small-diameter, noxious muscle afferent nerves (GIII, A $\delta$, ergoreceptors). It is experienced as a strong, painful pulsating sensation which is often accompanied by muscle twitching.

HF TENS was shown to be more effective for pain relief than placebo TENS in PD patients. ${ }^{6,7,10,17-19}$ By contrast, LF TENS was as efficient as placebo TENS or pills for pain relief. ${ }^{4}$

HF was preferred over LF when pain relief was reported as a dichotomous variable, ${ }^{4}$ but when the pain relief was measured by a visual analog scale, there were no significant differences, although there was a trend toward greater pain relief with HF TENS. ${ }^{17}$ However, one study reported a significant difference in favor of LF TENS for the number of analgesic tablets taken in addition to the treatment. ${ }^{4}$ The results should be interpreted with 


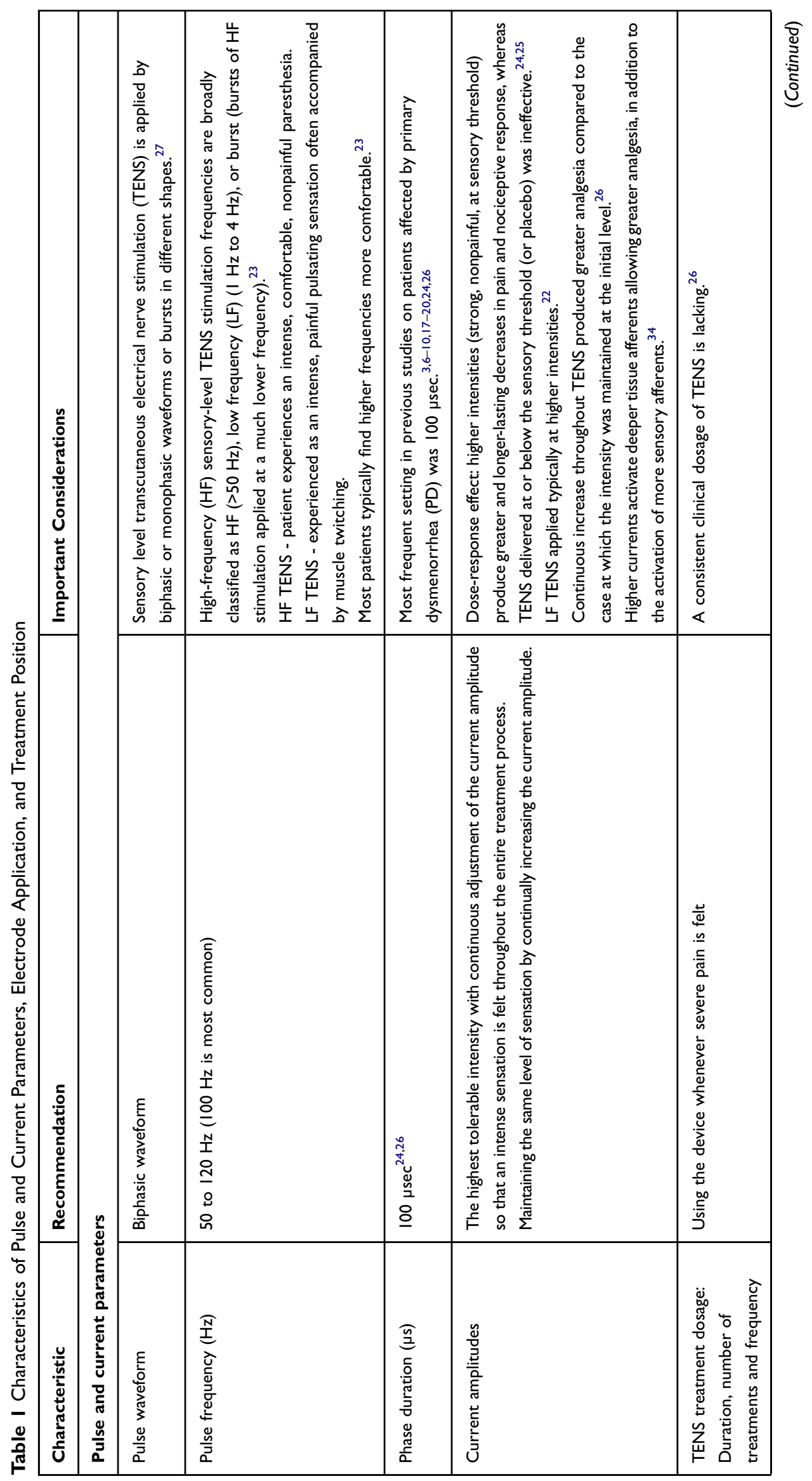




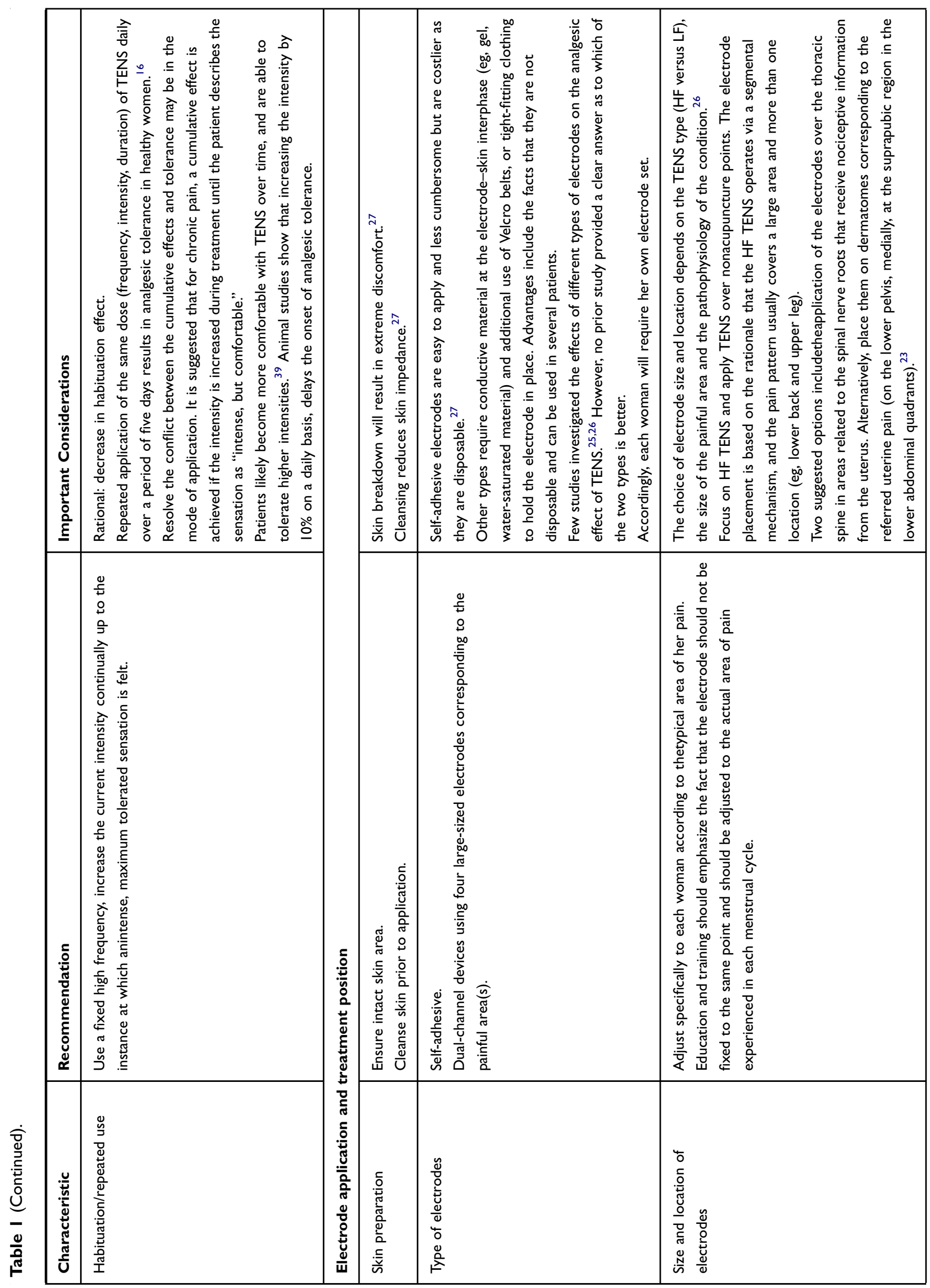




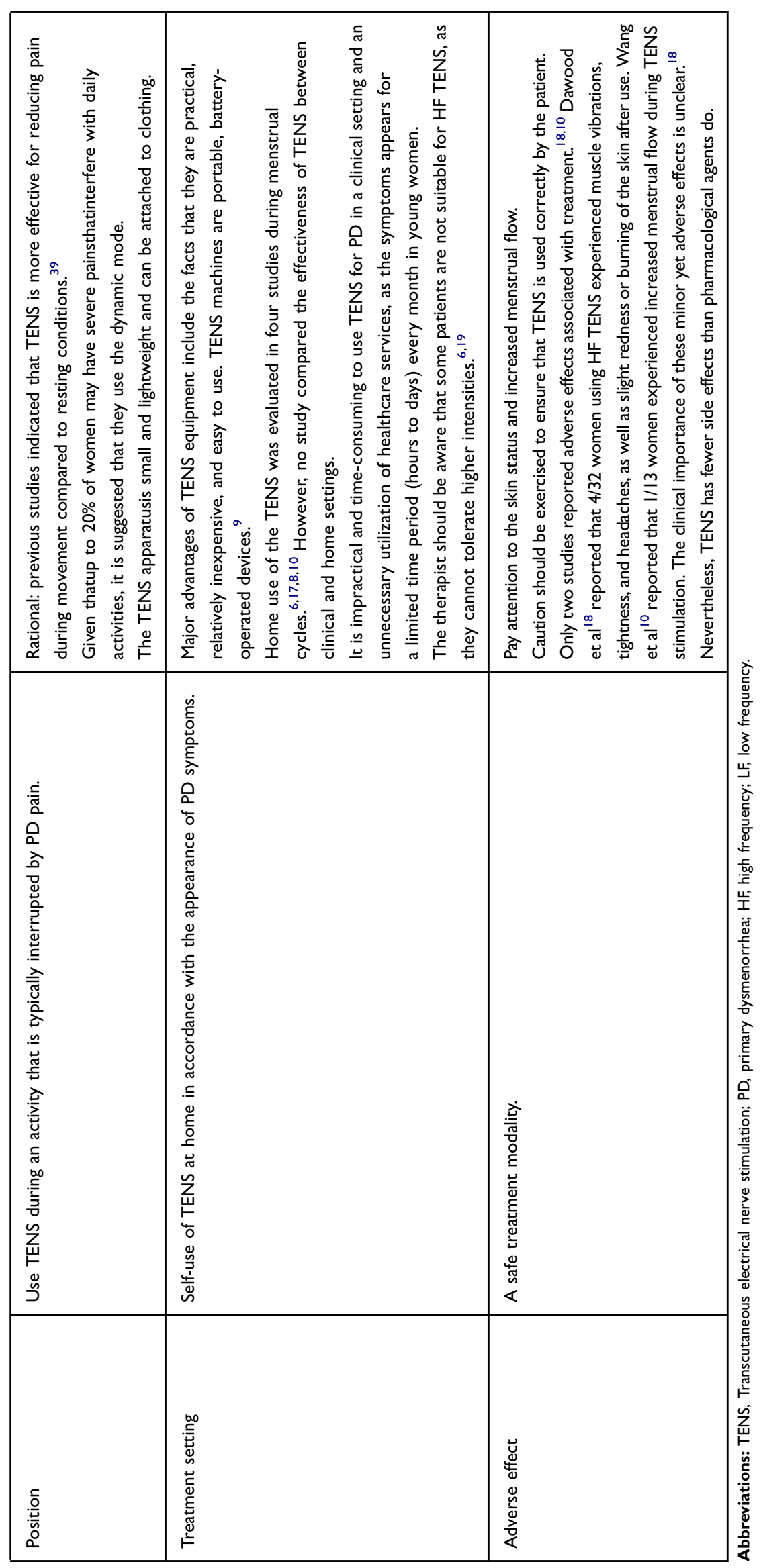


TENS treatment and adjustment should be scheduled when pain is reported

Education and explanation about the nature of the condition and providing ways of coping

Primary setting and adjustment of appropriate current parameters by the therapist in the clinic and training for effective and safe self-use

Promote continuous self-use at home and during daily activities

Consider reciprocal interaction owing to the consumption of additional opioids

Self-adhesive electrodes

High frequency $50-120 \mathrm{~Hz}$
Pulse width - 100

Biphasic waveform
Locations of the electrodes: over the area of pain in each menstrual cycle

Intensity: Gradually increase to the highest tolerable level, or at least until an intense but comfortable sensation is achieved. Maintain the same level of sensation throughout the TENS application

Provide safety instructions.

Special safety concerns owing to physiological and personality issues.

Use TENS whenever severe pain is felt

Figure I Summary of recommendations for transcutaneous electrical nerve stimulation (TENS) in primary dysmenorrhea.

caution as only two studies compared the efficiency of HF TENS to LF TENS in PD. ${ }^{17,19}$

The choice between HF or LF TENS also depends on the consumption of pharmacological agents, in particular analgesics, owing to reciprocal interactions. ${ }^{23}$ If a patient is taking opioids, HF TENS will likely be more effective, as LF works through the same pathway as the opioids, which may reduce its positive effects. ${ }^{23}$ In summary, HF TENS is recommended for treating women with PD (for details see Table 1 and Figure 1).

\section{TENS Stimulation Intensity}

The intensity of the stimulation is one of the most critical factors affecting the TENS efficacy. ${ }^{15,23}$ Studies have shown greater and longer-lasting decreases in pain and nociceptive response when TENS is applied at sensory thresholds with higher intensities/current amplitudes in which the individuals reported that the sense was significantly intense noticeable but nonpainful.
By contrast, TENS is not effective when applied at or below the sensory threshold (or placebo). ${ }^{24,25}$

The degree of hyperalgesia is significantly correlated with the current amplitude. Previous studies of the doseeffect response for TENS in PD could not be compared comprehensively owing to the variations in intensity and missing information.

The recommendation is to apply the TENS at the highest tolerable intensity ${ }^{15,23-25}$ while adjusting the current amplitude in a continuous manner so that its presence will be noticeable throughout the treatment ${ }^{15,23,25}$ (for details see Table 1 and Figure 1).

Citing safety concerns, some authors advised setting the stimulation intensity to the individual's comfort level and not to the highest tolerable level, although it has been shown that the highest tolerable level resulted in greater pain relief. As women with PD are typically healthy and without sensory deficits, we advise clinicians to encourage the users to increase the intensity of the TENS until it reaches the highest tolerable level. However, safety instructions should instruct 
users with special concerns owing to physiological or personality issues to pay special caution.

\section{Duration, Number, and Frequency of Treatments}

The optimal recommended dose, including the number, duration, and frequency of treatments was not uniform. Variations in each of the three dosage components did not allow comparisons among protocols used in previous studies. The number of treatments ranged from one $\mathrm{e}^{3,7,9,10,19,20}$ to several. ${ }^{5,6,8,17,18}$ The duration lasted from 20 mins up to the continuous application for $8 \mathrm{hrs}$. Further studies are warranted to create an evidence base for recommendations on TENS dosage for women with PD. However, we recommend use of the device whenever severe pain is felt for as long as needed, but $20-30$ mins is probably the minimum effective time. ${ }^{26,27}$

\section{Development of Habituation and Tolerance in Repeated Use of TENS}

As PD is experienced on several days every month and may last for years, habituation, tolerance, and repeated use of TENS should be considered

\section{Habituation}

Habituation is a decreased response to a repeated stimulus. ${ }^{27}$ The rate of habituation can be reduced by modulating one of the current parameters, usually the frequency or the amplitude. ${ }^{28}$ The effectiveness of frequency modulation is questionable. $^{23}$ By contrast, experimental and clinical studies demonstrated that increasing pulse amplitudes during TENS increased the analgesic effect. ${ }^{25,29}$ There is no evidence regarding the effects of frequency or intensity modulation among the PD population. However, based on current evidence, we recommend the use of a fixed HF, while continually increase the current intensity to an intense, maximum tolerated sensation, and maintain the sensation during the treatment session.

\section{Repeated Use}

Maintenance over time of the positive effect (accumulated effect) of the pain reduction following repeated usage have been reported, eg, in an application of TENS for five days of the week for four weeks in patients with knee osteoarthritis. ${ }^{30}$

Sluka et $\mathrm{al}^{21}$ explained the accumulation effect based on "the hypothesis that repeated TENS causes rebooting of one or more of the sensitization processes that results in a cumulative and longer-lasting TENS effect" (page $1398 \mathrm{in}^{21}$ ).

In addition, the accumulated effect can be explained as an accompanying consequence to decreased levels of pain that results in a greater physical activity level. Higher physical activity levels may maintain the pain relief that is achieved by the application of TENS.

Women who suffer from PD during all of their menses with negative effects on their activities and quality of life can use TENS over time (a few days each month). No studies examined the cumulative effect of TENS on PD, which differs from other chronic pain conditions as it can be referred to a time scale of days or successive months.

However, repeated use of the same dose of HF or LF (intensity, pulse duration) TENS on a daily basis can produce analgesic tolerance, which may decrease its positive effects. ${ }^{21}$ Decline in the hypoalgesia (measured by the pressure-pain threshold) have been demonstrated in painfree participants by the fourth or fifth day following daily usage of TENS in equal dose (frequency, intensity, pulse and treatment durations). ${ }^{31}$

The mode of application may help resolve the conflict between the cumulative effects and tolerance. It has been suggested that for chronic pain, a cumulative effect without the appearance of analgesic tolerance is achieved only if the current intensity is not fixed and there is continuous increase up to the state at which a clear intense sensation of the current is experienced but without the sense of pain. This increase is possible as the user gets accustomed to the current intensity over time, which enables further increases of the intensity to higher levels of tolerance. ${ }^{21}$ This suggestion is based on animal studies that showed that delayed onset of the analgesic tolerance can be achieved by applying continuous daily increases of the intensity by $10 \%{ }^{32}$ However, there is not a full understanding of the tolerance mechanisms in chronic pain owing to the intricacy of the neuronal processing alteration in these patients. ${ }^{23}$ Accordingly, owing to the lack of knowledge on the effects of repeated TENS over time in chronic pain as well as in the PD population, ${ }^{23}$ we recommend that women who use TENS over a long period should be monitored to detect the development of tolerance.

\section{Long-Term Usage}

Only Lauretti et $\mathrm{al}^{6}$ reported self-use of TENS for three months in the PD population, but they did not evaluate the effectiveness of the treatment over time. Most of the conducted "controlled clinical trials examined the TENS 
efficacy with a single treatment or for periods up to a few weeks" (page $1399 \mathrm{in}^{21}$ ). Previous studies that focused on long-term usage (defined as usage for periods of $\geq 6$ months) of TENS by subjects with chronic pain had shown a significant reduction in the consumption of analgestic medication, ${ }^{33-35}$ diminished usage of physical/ occupational therapy services, ${ }^{33,34}$ and increased physical activity and participation. ${ }^{34}$ Oosterhof et $\mathrm{al}^{36}$ demonstrated that the positive analgesic effect of TENS and sham TENS in patients with chronic pain is similar and is maintained over a period of one year.Accordingly, owing to the lack of data, there are requirements for performing randomized control studies to explore the effect of TENS efficacy in the long-term (months or years) compared with a placebo in the PD population.

\section{Interactions with Pharmacological Agents}

Previous results pointed on the potential interactions of opioid drugs with TENS owing to the mutual mechanism of activation of opioid receptors in the central nerve system. ${ }^{21}$ It is possible that the inefficiency of TENS is owing to the competition of the opioid receptors with opioid drugs. The frequency of TENS has demonstrated that it affects the type of receptors that are active, whereby LF TENS (1-10 Hz) activates mu-opioid receptors, while HF TENS $(50-150 \mathrm{~Hz})$ activates delta-opioid receptors. ${ }^{23}$

Leonard and colleague ${ }^{37}$ demonstrated that LF TENS had a positive effect only in patients who did not take opioids, and that there was no effectiveness in opioidtreated patients. However, HF TENS also demonstrated a positive effect in the opioid-treated patients.

As the main treatment option for PD is pain reliever pills, it would be interesting to compare the efficiency of TENS to pills. Three studies ${ }^{6,7,18}$ examined the use of analgesics in addition to TENS or compared these two treatment options. Dawood ${ }^{18}$ found that ibuprofen alone provided better pain relief than did active and sham TENS. Using HF TENS, the amount of ibuprofen consumed for up to $12 \mathrm{~h}$ was reduced, and significantly delayed the need for additional administration. In addition, application of TENS along with consumption of ibuprofen provided better pain reduction compared to TENS alone. Lundeberg et $\mathrm{al}^{19}$ stated that it appears that HF TENS is more effective than verapamil for pain relief and that HF TENS evoked almost the same effect as naproxen. However, the results should be interpreted with caution, as only descriptive data have been published, without statistical analyses. Lundeberg et al ${ }^{19}$ also examined six participants who had experienced pain relief with both HF and LF TENS, as the compound effect of the prescription and application of both treatment options. Accordingly, in four individuals, the supplement of intravenous naloxone hydrochloride solution to LF TENS abolished the positive effect of pain reduction gained by the LF TENS, whereas in the same individual the supplement did not alter the positive effect of HF TENS.

This may support findings from previous studies which suggested that the action mechanism of pain decrease evoked by LF TENS is different from HF (endogenous opioids versus nonopioid pathways, respectively) and mediates that evoked by HF TENS, whereas pain relief obtained with LF TENS appeared to be involved also. Yet, the reported study included very few subjects. ${ }^{4}$

\section{Conclusions}

PD has a negative effect on almost every facet of a woman's life for a few days each month over a period of multiple years. Managing PD with pharmacological agents is associated with considerable undesirable side effects. The major advantages of TENS are that is it is an easy-to-use, safe, portable, battery-operated, and relatively inexpensive device. The action mechanism of TENS is based on the enhancement of the endogenous inhibition and the suppression of central excitability.

The evoked results of previous studies demonstrated the positive effects of TENS in reducing pain and related PD symptoms, the improvement of the quality of life, and the decreased use of additional analgesics. Although these studies were associated with some flaws and limitations in their methodological quality and therapeutic validity, herein, recommendations and considerations for the effective use of TENS for managing PD were outlined. Regular, continuous self-administration in accordance with the appearance of PD symptoms is suggested after the initial patient education and setting, and after the adjustment of the TENS parameters by the therapist. Electrode placement should not be fixed at one point but should be moved and placed on the painful area(s). A HF (100 Hz) is suggested owing to the proven effectiveness with PD and the comfortable, convenient use. HF TENS may be more effective for people taking opioids. LF TENS may be considered in special cases of regular analgesic consumption. The most important parameter is the current intensity, which should be set to a nonpainful, maximal tolerable 
intensity level. The intensity should be increased continually throughout the treatment session to maintain an intense sensation and avoid habituation. TENS is probably ineffective for women who cannot reach and maintain increased current intensities.

Additional higher quality research studies are required to expand the current knowledge on the applications of TENS for PD based on large sample datasets. Adequate dosing (number of treatments, duration, and frequency) in managing PD should be explored. Additional investigations should determine if a "wash-out" period is required for women who use TENS regularly during menstruation. Using the Initiative on Methods, Measurement, and Pain Assessment in Clinical Trials (IMMPACT) guidelines as outcome measures in clinical trials on pain ${ }^{38}$ will further assist in the adequate comparison of TENS with other treatment options. Furthermore, the evaluated outcomes should be expanded beyond the resting pain to include activity levels, function, and quality of life.

\section{Disclosure}

The authors report no conflicts of interest in this work.

\section{References}

1. Lefebvre G, Pinsonneault O, Antao V, et al. Primary dysmenorrhea consensus guideline. Can J Obstet Gynaecol. 2005;27(12):1117-1146.

2. Kannan P, Chapple CM, Miller D, Claydon LS, Baxter GD. Menstrual pain and quality of life in women with primary dysmenorrhea: rationale, design, and interventions of a randomized controlled trial of effects of a treadmill-based exercise intervention. Contemp Clin Trials. 2015;42:81-89. doi:10.1016/j.cct.2015.03.010

3. Milsom I, Hedner N, Mannheimer C. A comparative study of the effect of high-intensity transcutaneous nerve stimulation and oral naproxen on intrauterine pressure and menstrual pain in patients with primary dysmenorrhea. Am J Obstet Gynecol. 1994;170(1):123-129. doi:10.1016/s0002-9378(94)70396-5

4. Proctor M, Farquhar C, Stones W, He L, Zhu X, Brown J. Transcutaneous electrical nerve stimulation and acupuncture for primary dysmenorrhoea. Cochrane Database Syst Rev. 2002;1:CD002123.

5. Bai H-Y, Bai H-Y, Yang Z-Q. Effect of transcutaneous electrical nerve stimulation therapy for the treatment of primary dysmenorrheal. Med. 2017:96(36):e7959. doi:10.1097/MD.0000000000007959

6. Lauretti GR, Oliveira R, Parada F, Mattos AL. The new portable transcutaneous electrical nerve stimulation device was efficacious in the control of primary dysmenorrhea cramp pain. Neuromodulation. 2015;18(6):522-527. doi:10.1111/ner.12269

7. Parsa P, Bashirian S. Effect of transcutaneous electrical nerve stimulation (TENS) on primary dysmenorrhea in adolescent girls. $J$ Postgrad Med Inst (Peshawar-Pakistan). 2013;27(3):326-330.

8. Schiøtz H, Jettestad M, Al-Heeti D. Treatment of dysmenorrhoea with a new TENS device (OVA). J Obstet Gynaecol. 2007;27(7):726-728. doi:10.1080/01443610701612805

9. Tugay N, Akbayrak T, Demirtürk F, et al. Effectiveness of transcutaneous electrical nerve stimulation and interferential current in primary dysmenorrhea. Pain Med. 2007;8(4):295-300. doi:10.1111/j.15264637.2007.00308.x
10. Wang SF, Lee JP, Hwa HL. Effect of transcutaneous electrical nerve stimulation on primary dysmenorrhea. Neuromodulation. 2009;12 (4):302-309. doi:10.1111/j.1525-1403.2009.00226.x

11. Kannan P, Claydon LS. Some physiotherapy treatments may relieve menstrual pain in women with primary dysmenorrhea: a systematic review. J Physiother. 2014;60(1):13-21. doi:10.1016/j.jphys.2013.12.003

12. Latthe P, champaneria R, Khan KS. Dysmenorrhoea. BMJ Clin Evid. 2011;21:2011.

13. Maybin JA, Critchley HO. Menstrual physiology: implications for endometrial pathology and beyond. Hum Reprod Update. 2015;21 (6):748-761. doi:10.1093/humupd/dmv038

14. Melzack R, Wall PD. Pain mechanisms: a new theory. Science. 1965;150(3699):971-979. doi:10.1126/science.150.3699.971

15. Vance CG, Dailey DL, Rakel BA, Sluka KA. Using TENS for pain control: the state of the evidence. Pain Manag. 2014;4(3):197-209. doi: $10.2217 / \mathrm{pmt} .14 .13$

16. Lewers D, Clelland JA, Jackson JR, Varner RE, Bergman J. Transcutaneous electrical nerve stimulation in the relief of primary dysmenorrhea. Phys Ther. 1989;69(1):3-9. doi:10.1093/ptj/69.1.3

17. Mannheimer JS, Whalen EC. The efficacy of transcutaneous electrical nerve stimulation in dysmenorrhea. Clin J Pain. 1985;1(2):75-84. doi:10.1097/00002508-198501020-00004

18. Dawood MY, Ramos J. Transcutaneous electrical nerve stimulation (TENS) for the treatment of primary dysmenorrhea: a randomized crossover comparison with placebo TENS and ibuprofen. Obstet Gynecol. 1990;75(4):656-660.

19. Lundeberg T, Bondesson L, Lundströom V. Relief of primary dysmenorrhea by transcutaneous electrical nerve stimulation. Acta Obstet Gynecol Scand. 1985;64(6):491-497. doi:10.3109/00016348509156727

20. Neighbors LE, Clelland J, Jackson JR, Bergman J, Orr JJ. Transcutaneous electrical nerve stimulation for pain relief in primary dysmenorrhea. Clin J Pain. 1987;3(1):17-22. doi:10.1097/00002508198703010-00004

21. Sluka KA, Bjordal JM, Marchand S, Rakel BA. What makes transcutaneous electrical nerve stimulation work? Making sense of the mixed results in the clinical literature. Phys Ther. 2013;93 (10):1397-1402. doi:10.2522/ptj.20120281

22. Kitchen S, Bazin S, Bellis E. Electrotherapy: Evidence-Based Practice. 11th ed. London, UK: Churchill Livingstone; 2002.

23. Sluka KA, Walsh D. Transcutaneous electrical nerve stimulation: basic science mechanisms and clinical effectiveness. J Pain. 2003;4 (3):109-121. doi:10.1054/jpai.2003.434

24. Bjordal JM, Johnson MI, Ljunggreen AE. Transcutaneous electrical nerve stimulation (TENS) can reduce postoperative analgesic consumption. A meta-analysis with assessment of optimal treatment parameters for postoperative pain. Eur J Pain. 2003;7(2):181-188. doi:10.1016/S1090-3801(02)00098-8

25. Moran F, Leonard T, Hawthorne S, et al. Hypoalgesia in response to transcutaneous electrical nerve stimulation (TENS) depends on stimulation intensity. J Pain. 2011;12(8):929-935. doi:10.1016/j. jpain.2011.02.352

26. Cameron MH. Physical Agents in Rehabilitation: From Research to Practice. Elsevier Health Sciences; 2012.

27. Robinson AJ. Clinical Electrophysiology: Electrotherapy and Electrophysiologic Testing. Lippincott Williams \& Wilkins; 2008.

28. Spielholz N, Nolan M. Conventional TENS and the phenomenon of accommodation, adaptation, habituation and electrode polarization. J Clin Electrophysiol. 1995;7:16-19.

29. Pantaleao MA, Laurino MF, Gallego NL, et al. Adjusting pulse amplitude during transcutaneous electrical nerve stimulation (TENS) application produces greater hypoalgesia. J Pain. 2011;12 (5):581-590. doi:10.1016/j.jpain.2010.11.001

30. Cheing GL, Hui-Chan CW, Chan K. Does four weeks of TENS and/ or isometric exercise produce cumulative reduction of osteoarthritic knee pain? Clin Rehabil. 2002;16(7):749-760. doi:10.1191/026921 5502 cr549oa 
31. Liebano RE, Rakel B, Vance CG, Walsh DM, Sluka KA. An investigation of the development of analgesic tolerance to TENS in humans. Pain. 2011;152(2):335-342. doi:10.1016/j.pain.2010.10.040

32. Sato KL, Sanada LS, Rakel BA, Sluka KA. Increasing intensity of TENS prevents analgesic tolerance in rats. $J$ Pain. 2012;13 (9):884-890. doi:10.1016/j.jpain.2012.06.004

33. Chabal C, Fishbain DA, Weaver M, Heine LW. Long-term transcutaneous electrical nerve stimulation (TENS) use: impact on medication utilization and physical therapy costs. Clin J Pain. 1998;14 (1):66-73. doi:10.1097/00002508-199803000-00010

34. Fishbain DA, Chabal C, Abbott A, Heine LW, Cutler R. Transcutaneous electrical nerve stimulation (TENS) treatment outcome in long-term users. Clin J Pain. 1996;12(3):201-214. doi:10.1097/00002508-19960900000008

35. Johnson M, Ashton C, Thompson J. Long term use of transcutaneous electrical nerve stimulation at Newcastle Pain Relief Clinic. J R Soc Med. 1992;85(5):267.
36. Oosterhof J, Wilder-Smith OH, de Boo T, et al. The long-term outcome of transcutaneous electrical nerve stimulation in the treatment for patients with chronic pain: a randomized, placebo-controlled trial. Pain Pract. 2012;12(7):513-522. doi:10.1111/j.1533-2500.2012.00533.x

37. Léonard G, Cloutier C, Marchand S. Reduced analgesic effect of acupuncture-like TENS but not conventional TENS in opioid-treated patients. J Pain. 2011;12(2):213-221. doi:10.1016/j. jpain.2010.07.003

38. Turk D, Dworkin R, Burke L, et al. Initiative on methods, measurement and pain assessment in clinical trials. Developing patient-reported outcome measures for pain clinical trials: IMMPACT recommendations. Pain. 2006;125(3):208-215. doi:10.1016/j.pain.2006.09.028

39. Dailey DL, Rakel BA, Vance CG, et al. Transcutaneous electrical nerve stimulation reduces pain, fatigue and hyperalgesia while restoring central inhibition in primary fibromyalgia. PAIN®. 2013;154 (11):2554-2562. doi:10.1016/j.pain.2013.07.043

\section{Publish your work in this journal}

The International Journal of Women's Health is an international, peerreviewed open-access journal publishing original research, reports, editorials, reviews and commentaries on all aspects of women's healthcare including gynecology, obstetrics, and breast cancer. The manuscript management system is completely online and includes a very quick and fair peer-review system, which is all easy to use. Visit http://www.dovepress.com/testimonials.php to read real quotes from published authors. 